\title{
Meridional Circulation with Latitude Bands of Long-Lived Cyclones in Jupiter's Convective Atmosphere
}

\author{
Hans G. Mayr ${ }^{1}$, Kwing L. Chan² \\ ${ }^{1}$ NASA Goddard Space Flight Center, Greenbelt, USA (Retired) \\ ${ }^{2}$ Macau University of Science and Technology, Macau, China \\ Email: hmayr2@verizon.net, klchan@must.edu.mo
}

How to cite this paper: Mayr, H.G. and Chan, K.L. (2021) Meridional Circulation with Latitude Bands of Long-Lived Cyclones in Jupiter's Convective Atmosphere. International Journal of Astronomy and Astrophysics, 11, 392-405.

https://doi.org/10.4236/ijaa.2021.113018

Received: February 3, 2021

Accepted: September 10, 2021

Published: September 13, 2021

Copyright $\odot 2021$ by author(s) and Scientific Research Publishing Inc. This work is licensed under the Creative Commons Attribution International License (CC BY 4.0).

http://creativecommons.org/licenses/by/4.0/

\begin{abstract}
Mayr et al. [1] proposed that the vertical velocities in the global scale meridional circulation can produce distinct latitude bands where Jovian vortices like the white and brown are observed, and we present here a brief review of the mechanism. The observed life times of the ovals are much longer than the estimated spin-down times, which indicates that the vortices must be sustained through the release of internal energy. Like Jupiter's Great Red Spot (GRS), the white/brown ovals are treated like terrestrial hurricanes or cyclones, which are generated by convection. The planetary energy Jupiter emits is transferred by convection, and under this condition the upward motions in the meridional circulation, around the equator for example, release energy from below and decrease the convective instability to suppress the formation of cyclones. But the downward motions in the circulation, near $20^{\circ}$ latitude for example, carry energy down so that the convective instability is amplified to produce a dynamical environment that is favorable for the development of cyclones like the GRS and white/brown ovals. This picture is supported by an analysis of results from a numerical model of Jupiter's alternating jets (Chan and Mayr [2]). Generated by alternating vertical winds in the meridional circulation, the vertical temperature variations reveal distinct latitude bands with enhanced convective instability, most prominent at high latitudes where long-lived circumpolar cyclones are observed from the Juno spacecraft.
\end{abstract}

\section{Keywords}

Jovian Vortices Like Great Red Spot, Convection, Latitudinal Vortex Stratification, Meridional Flow, Numerical Model Results 


\section{Introduction}

A gaseous planet, Jupiter emits more than twice as much energy as it receives from the Sun. The planetary energy from the interior is carried by convection to the top of the atmosphere, where it is radiated away from the stable region around the clouds. Jupiter's vortices like the Great Red Spot (GRS) and the alternating zonal jets are mainly observed in the cloud layer (e.g., Smith et al. [3] [4]; Mitchell et al. [5]; Simon-Miller et al. [6]; Porco et al. [7]; Sánchez-Lavega et al. [8]; Galanti et al. [9]; Tabataba-Vakili et al. [10]), and model simulations have been confined to that region (e.g., Marcus [11], Marcus et al. [12]; Marcus et al. [13]; Showman et al. [14]; Showman [15]). On a different path, recent numerical models have been showing that convective energy transport below the clouds can generate the alternating jets (e.g., Mayr et al. [16]; Ingersoll et al. [17]; Zhang and Schubert [18]; Christensen [19]; Aurnou and Olson [20]; Heimpel and Aurnou [21]; Chan and Mayr [2]). And Chan and Mayr [22] produced Jovian type vortices purely by convection, without imposing artificial shears and flow fields in support of the mechanism Kuiper [23] had proposed, linking the GRS to the terrestrial hurricane.

Based on the theory developed by Charney and Eliassen [24] and Ooyama [25], the terrestrial hurricane is generated by the so-called Conditional Instability of Second Kind (CISK), which requires that the lower layer in the Earth's tropical atmosphere is convective and conditionally unstable, i.e., unsaturated and wet-super adiabatic. When a vortex develops, convergence of the horizontal flow brings moisture into the center and forces ascension with release of latent energy, and this process in turn refuels the motions.

Considering that the observed horizontal dimension of the planetary vortex can reach as an upper limit the Rossby diameter of deformation (Holton [26]), the depths, h, and spin-down times, $\tau$, can be estimated (Holton [27]) to produce $\mathrm{h}=400 \mathrm{~km}$ and $200 \mathrm{~km}$, and $\tau=1.6$ years and 4 months for the GRS and the white/brown ovals, respectively (Mayr et al. [1]). The long life time of the GRS, discovered centuries ago, indicates that it is rooted deep in the convective region of Jupiter's atmosphere, and high-precision gravity measurements with the Juno mission are exploring the depth of the vortex down to more than 300 $\mathrm{km}$ (Gilanti et al. [9]). The white and brown ovals also have live times exceeding the spin-down times, which suggests that they originate well below the clouds. These long-lived vortices are observed organized along latitude bands, and Mayr et al. [1] propose that the downward motions in the global-scale meridional circulation can produce regions with amplified convective instability, varying with latitude, where the dynamical conditions are favorable for generating and sustaining the Jovian vortices.

\section{Vortex Generations}

Jovian vortices and terrestrial hurricanes have in common that they produce the same flow pattern at the top of the atmosphere. This is seen for example in some 
of the earliest close-up observations of the Great Red Spot (GRS) and white ovals that were taken from Voyager I in March 1979 (Smith et al. [3] [4]); and Skylab took pictures of hurricane Irah on September 24, 1973. The sense of rotation is anti-cyclonic, i.e., counter-clockwise for the GRS that is located in the southern hemisphere $\left(20^{\circ} \mathrm{S}\right)$, and clockwise for Irah that appeared in the northern hemisphere $\left(18^{\circ} \mathrm{N}, 107.8^{\circ} \mathrm{W}\right)$. Under the influence of the Coriolis force, anti-cyclonic motions are sustained by enhanced pressure and related temperature variations inside the vortex.

In the case of the terrestrial hurricane or tropical cyclone, a low pressure system is formed near the ground to produce a cyclonic flow pattern. The spiraling winds are drawn into the center of the hurricane, and rising motions with convection fuel the high pressure system above, which generates the anti-cyclonic motions at higher altitudes.

For the GRS in contrast the picture is not that clear, because the measurements have been limited to the convectively stable atmospheric layer around the clouds. Figure 1(a) shows the vertical temperature variations inside and outside the GRS, which were derived from infrared measurements on Voyager I (Hanel et al. [28]). Below the tropopause, the temperatures increase towards lower altitudes with gradients close to super adiabatic; convection becomes important. Within the limited altitude range of observations, there is no indication of enhanced temperatures inside the GRS. To produce the anti-cyclonic rotation of the vortex, the pressure must be built up by temperature variations further down in the convective region of the troposphere, illustrated in Figure 1(b). In analogy with the terrestrial hurricane mechanism, it is proposed that the upward
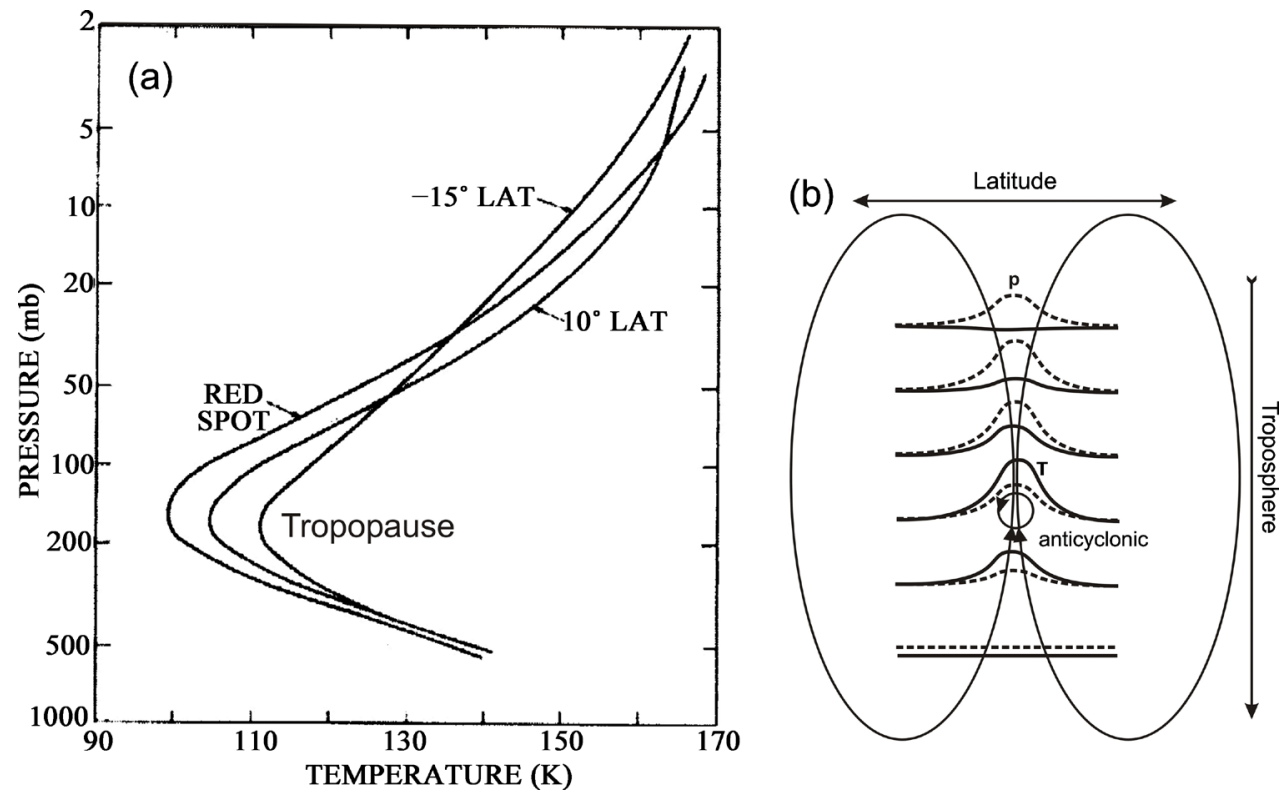

Figure 1. (a) Vertical temperature variations inside and outside the GRS derived from infrared measurements on Voyager 1, taken from Hanel et al. [28]. (b) Proposed schematic cross sections of temperature (solid line) and pressure (dashed line) variations in the convective troposphere. (Figure taken from Mayr et al. [1].) 
motions inside the GRS transfer energy up from below to increase the temperature, which in turn is building up the pressure. Due to expansion from below, the pressure continues to be enhanced at higher altitudes to maintain the anti-cyclonic vortex near the clouds. Infrared measurements from Voyager I (Hanel et al. [28]) show traces of water in the troposphere of Jupiter. Condensation of water vapor with release of latent energy can contribute to generate the long-lived Jovian vortices.

\section{Latitudinal Vortex Stratification}

The alternating zonal jets of Jupiter are in geostrophic balance, produced by latitudinal pressure and temperature variations. Evidence of that is seen for example in Voyager infrared measurements (e.g., Hanel et al., [28] [29]) that show considerable structure in the temperature at the 0.8 bar pressure level. Plateaus are observed, which are formed by temperatures that decrease abruptly towards higher latitudes near $20^{\circ}$ and $60^{\circ}$. Moreover, there is some indication that the zonal-mean temperature at the 0.15 bar level has a minimum near $20^{\circ}$ latitude.

The latitudinal temperature/pressure variations are generated by the internal convective energy source and in part by solar heating. The energy is mainly dissipated by the multi-cellular meridional circulation (Mayr and Harris [30]; Mayr et al. [16]) which is involved (Mayr et al. [1]) in the formation of the latitude bands where the long lived Jovian vortices are mainly observed.

For the purpose of this discussion, it is helpful to present in simplified form the energy equation for the zonal-mean variations of the circulation

$$
\alpha T+W c_{p}(\partial T / \partial z+\Gamma)=Q
$$

where $\alpha$ is the effective cooling coefficient due to turbulent heat conduction and radiation, $\mathrm{T}$ temperature, $\mathrm{W}$ vertical velocity of meridional circulation, $c_{p}$ specific heat at constant pressure, $z$ altitude, $\Gamma$ adiabatic temperature lapse rate, and $Q$ the energy source like solar heating. With stability $S=(\partial T / \partial z+\Gamma)$, the atmosphere is convectively stable, $S>0$, or unstable, $S<0$.

With Jupiter's internal energy source, the average global stability, $S$, is small. Thus relatively small changes in the temperature, $\Delta T$, can significantly affect the latitudinal variations of the stability $(S+\Delta S)$.

In the convective troposphere $(S<0)$, the prevailing upward motions in the meridional circulation $(W>0)$, around the equator for example, supply from below energy to the ambient medium, which increases the temperature and stability $(\Delta S>0)$, illustrated in Figure 2(a). Energy that otherwise may contribute to fuel a disturbance is transported to higher altitudes, and the consequence is that localized disturbances are suppressed. It is less likely that hurricane like vortices develop in the updraft regions of the large scale meridional circulation.

On the other hand, near $20^{\circ}$ latitude for example, where downward motions occur in the meridional circulation, energy is removed from the surrounding ambient medium and is transported to lower altitudes. The temperature decreases, and the atmosphere becomes less stable or more convective $(\Delta S<0)$, 


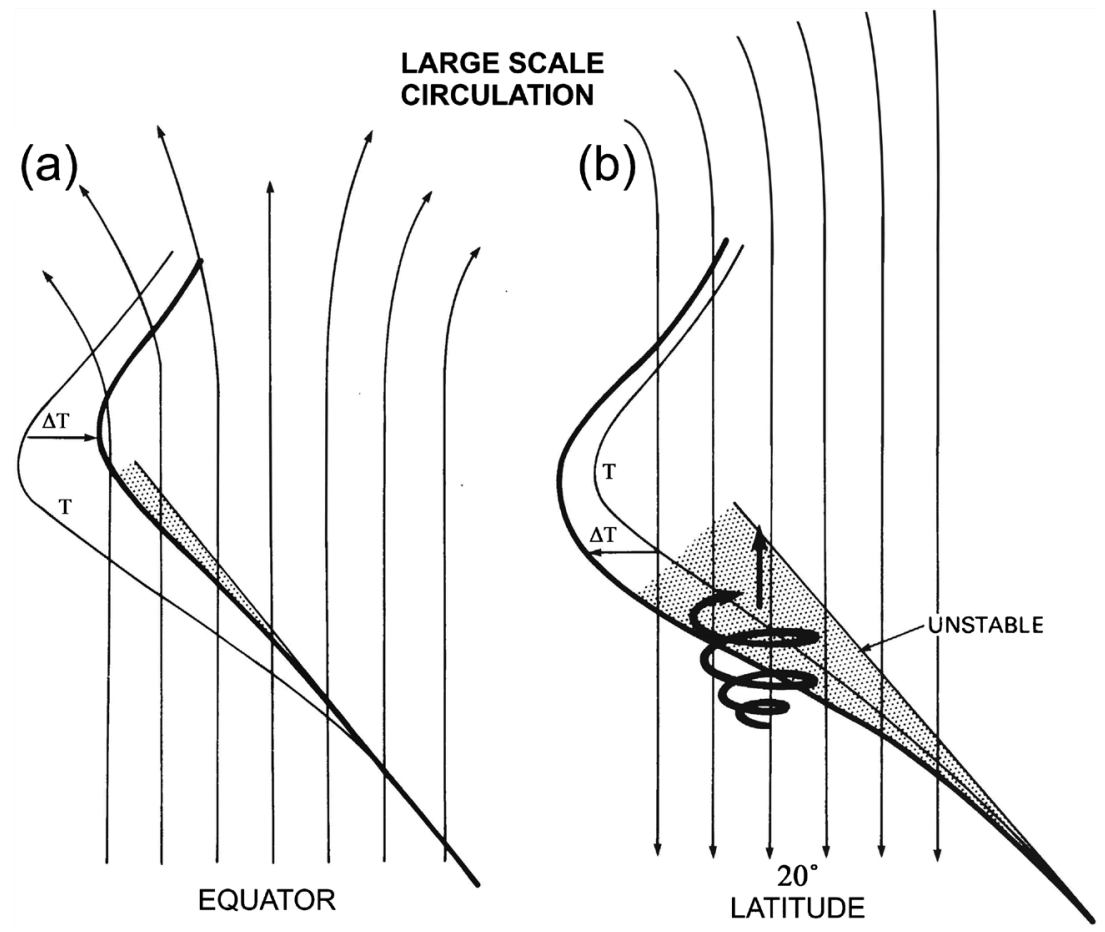

Figure 2. Schematic illustration of the vertical temperature variations, produced by the large scale meridional circulation (thin lines). On the left side (a) is shown the up-draft region around the equator for example, where the temperature increases, $\Delta \mathrm{T}>0$, and the stability, $\Delta S>0$. Hurricanes like vortices are less likely to develop in this region. On the right side (b) is shown a region near $20^{\circ}$ latitude for example, where the meridional circulation produces downward motions. The temperature and stability then decrease, $\Delta \mathrm{T}<$ $0, \Delta S<0$. Localized disturbances or vortices are more likely to develop. (Figure taken from Mayr et al. [1].)

illustrated in Figure 2(b). As a result, localized disturbances will be accelerated, and vortices can develop and be sustained. The Great Red Spot (GRS) and brown ovals are observed along latitude bands around $-20^{\circ}$ (Smith et al. [3]), and the latitudinal stratifications observed in the white ovals can also be produced by the downward motions in the multi-cellular meridional circulation.

Latitudinal cross sections of the prevailing temperature and zonal wind fields are illustrated in Figure 3. Away from the equator, the temperature (pressure) decreases, which can produce (assuming geostrophy) the eastward equatorial jet. Around $25^{\circ}$, the temperature variation reverses and can produce the westward jet. In between presumably, a temperature minimum lies, where the atmosphere is less stable, and hurricane like vortices can develop such as the GRS and white/brown ovals. The anticyclonic motions inside the Jovian hurricanes may be embedded in a zonal velocity field that is cyclonic, so that swirls and wake effects develop as illustrated in Figure 3.

The above discussion about the Jovian vortices in relation to the zonal circulation applies to the altitude regime where they are created. Figure 3 does not portray the conditions at higher altitudes near the top of the visible clouds where the observations come from. The GRS was observed $70^{\circ} \mathrm{W}$ and $100^{\circ} \mathrm{W}$ in system 


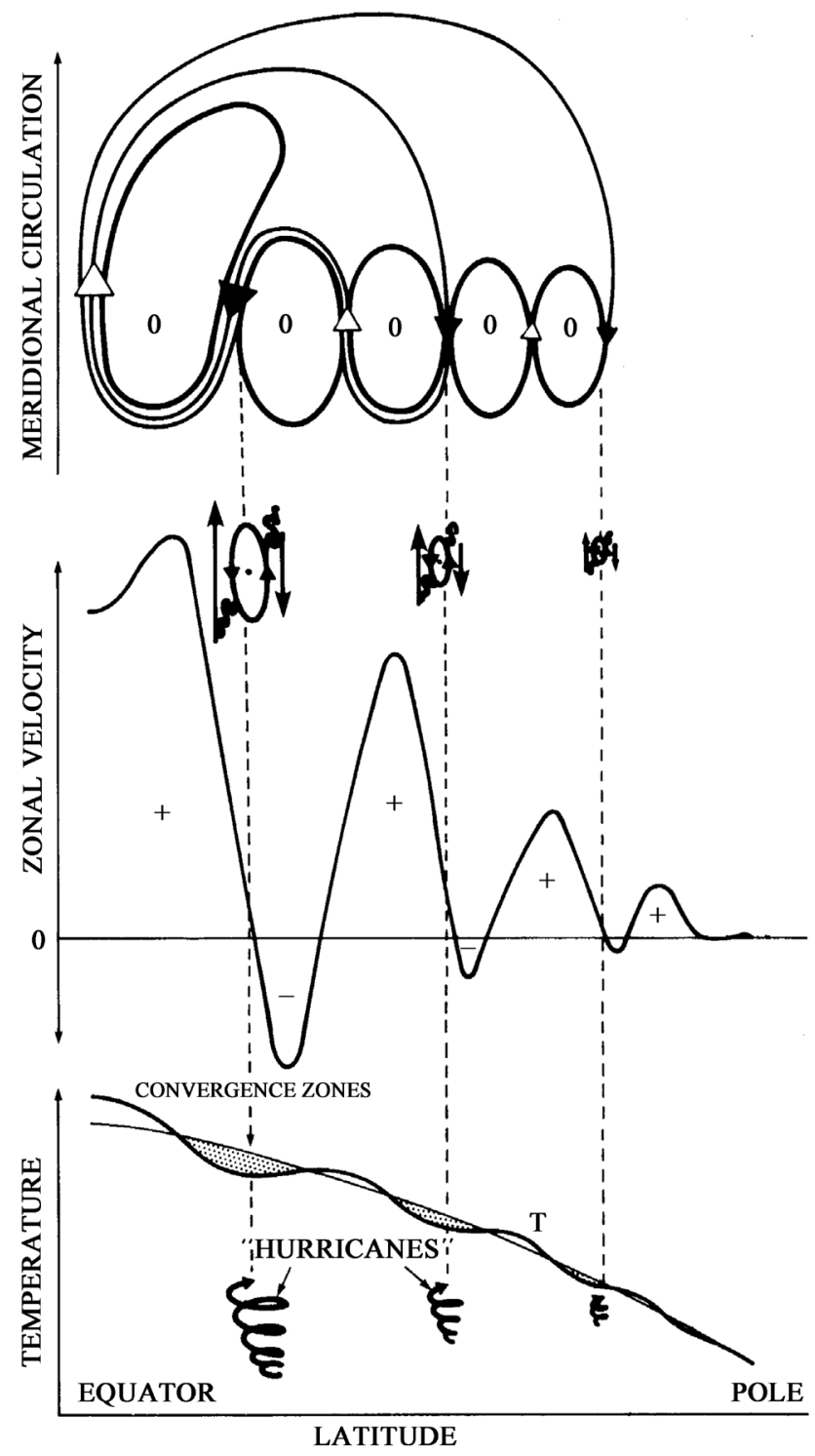

Figure 3. Illustration of the relationship between the multi-cellular meridional circulation (top), zonal circulation (middle), and relative temperature variations (bottom). Rising motions (light arrows) increase the temperature, illustrated in Figure 2(a), and the stability is enhanced (e.g. in the equatorial region). Downward motions (dark arrows) decrease the temperature, illustrated in Figure 2(b), and the stability is reduced (e.g. near $20^{\circ}$ latitude). In between, the temperature (pressure) falls off towards higher latitudes, driving eastward jets. Poleward of the temperature minimum, a westward jet can develop. The hurricane type vortex would tend to form in the shear region of the zonal velocity field in between. Due to the multicellular structure of the meridional circulation, this alternating pattern of upward and downward motions can produce alternating latitude bands, where Jovian type hurricanes are "forbidden" or "stimulated", respectively. (Figure taken from Mayr et al. [1].)

III during the Voyager I (1 February 1979) and Voyager II (23 May 1979) encounters, respectively. This produces a large 0.27 /day retrograde drift of the GRS within a short period of time (Figure 1 in Smith et al. [4]), which is consistent with the $100 \mathrm{yr}$ mean drift $\left(1.05 \times 10^{4} \mathrm{deg}\right.$ between 1850 and 1950). This drift direction is opposite to that of the observed zonal winds near the visible clouds 
and may indicate that the latitudinal structure in the prevailing circulation is changing significantly over the large depth of the GRS. Consistent with the pattern illustrated in Figure 3, the Voyager observations show that the white and brown ovals tend to move eastward in the direction of the zonal winds.

The Great Red Spot has been observed since its discovery by Casini in 1665, and this huge long-lived vortex is unique. Speculating about its creation, an enormous meteor might have plunged deep into the atmosphere of the southern tropical zone where the dynamical conditions are favorable for generating and sustaining Jovian hurricanes.

\section{Model Generated Latitude Bands of Instability}

The above discussed mechanism for the formation of latitude bands with hurricane type vortices is supported by numerical results from a spectral model that generates Jupiter's alternating zonal jets (Chan and Mayr [2]). The model is formulated with associated vector spherical harmonics for the numerical solution of the time dependent 3D nonlinear Navier Stokes equations (Chan et al. [31]). The spherical harmonics are truncated at 20 degrees (T20), and 68 radial grid levels cover the $430 \mathrm{~km}$ vertical depth of the atmosphere that is fully stratified and compressible. Stress-free and impenetrable boundary conditions are applied at the top and bottom of the $0.6 \%$ radial layer model domain. Jupiter's internal energy flux is applied but the smaller Solar input is ignored. The planetary energy is carried by convection over $95 \%$ of the layer and is emitted by radiative diffusion from a stable layer at the top. A uniform kinematic viscosity is adopted to dissipate the kinetic energy generated by convection.

In Figure 4 are shown the zonal-mean components of the zonal velocities (a) and relative temperature variations (b). Alternating winds are generated, and the velocities are within a factor of two of the observed values. In agreement with the Voyager observations (Smith et al. [6]), the model reproduces the large velocity peak at the equator and steep retrograde winds at $20^{\circ}$ latitudes. With Jupiter's internal energy source applied, the zonal winds are varying in the radial direction -in contrast to the Taylor column models (e.g., Christensen [19]; Aurnou and Olson [20]; Heimpel and Aurnou [21]) that are generated with unrealistic hyper-energetic energy source. In geostrophic balance, the temperature generated pressure variations with latitude produce the zonal wind velocities. The overall latitude pattern of the relative temperature variations is similar to that of the zonal winds, except for the region around the equator where the vanishing Coriolis force produces relatively small perturbations.

For the latitudinal variations of the meridional circulation the numerical results are presented in Figure 5. The vertical winds (a) highlight the multi-cellular pattern characteristic of Jupiter's circulation. The meridional winds (b) show converging and diverging velocity patterns with opposite directions in the opposite hemispheres. In common with the zonal wind pattern in Figure 4, a single circulation cell dominates around the equator, and multi-cellular circulation bands cover the latitudinal variations. Controlled by flow continuity, the 

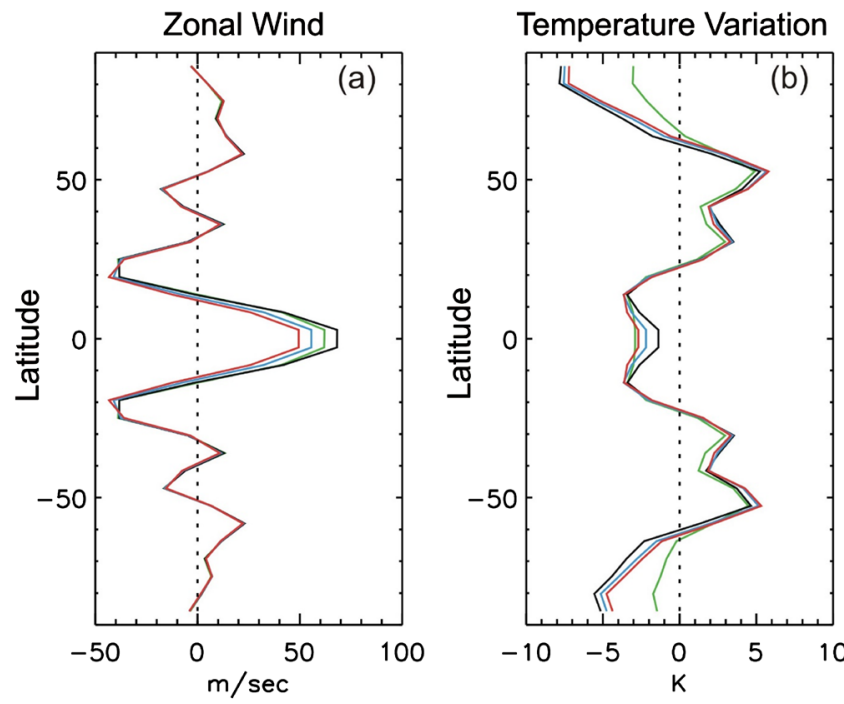

(b)

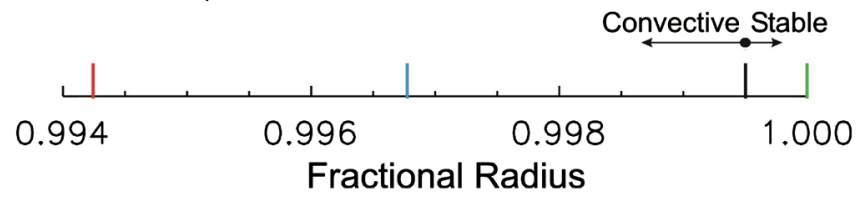

Figure 4. (a) Latitudinal variations of mean zonal winds (averaged in longitude) at altitudes indicated on a fractional (\%) radius scale. Top of domain, green; top of convection region, black; middle of convection region, blue; bottom of domain, red. Width of prograde equatorial jet agrees with observations, but $70 \mathrm{~m} / \mathrm{s}$ winds are a factor of 2 smaller than observed. (b) The relative temperature variations, larger than observed, are shown with collar scale identical to zonal winds (a). (Figures are taken from Chan and Mayr [2].)
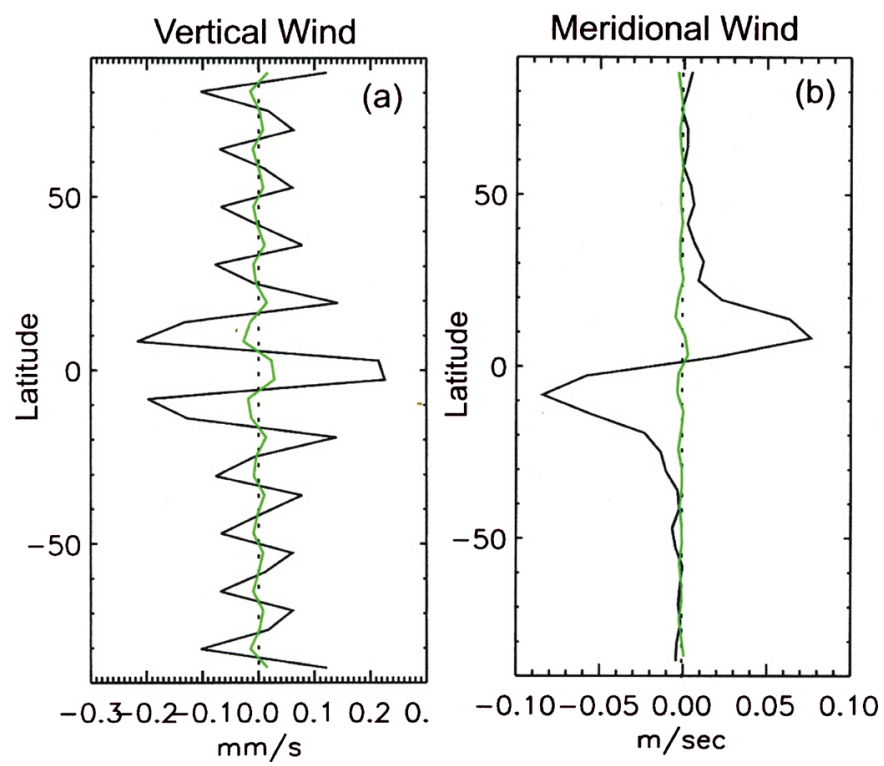

Figure 5. (a) Computed mean vertical winds ( $\mathrm{mm} / \mathrm{s}$ ) at top (black) and bottom (green) of convection region. Controlled in part by the short horizontal scale of Jupiter's circulation, the vertical winds are comparatively small. The latitudinal wind pattern is symmetric, with dominant upward velocity at the equator. (b) The larger meridional winds have opposite directions in the northern and southern hemispheres. Obeying flow continuity, the velocities are much larger at the top of the domain (black versus red/green). (Figures are taken from Chan and Mayr [32].) 
meridional winds are much larger at higher altitudes in the atmosphere where the background densities are much smaller compared to the lower portion. In contrast to the large rotational zonal winds, the smaller meridional winds have divergence, involved in generating the temperature and pressure variations that produce the zonal winds.

In Figure 6 are shown the computed temperature variations, $\Delta \mathrm{T}$, relative to the global average, T, taken from Chan and Mayr [2]. For the southern hemisphere, the temperatures are presented at different altitudes, identified with the underlying color code.

With the planetary energy source from the interior, the global-average atmosphere is convectively unstable, $S=(\partial T / \partial z+\Gamma)<0$ and the varying stability/instability across the globe, $\Delta S=(\partial \Delta T / \partial z+\Gamma)$, can be deduced from $d \Delta T$ (black-top - red-bottom) that varies with the vertical temperature gradient, $\partial \Delta T / \partial z$. Around the equator the model results show that the temperature increases with altitude, $d \Delta T>0(\partial \Delta T / \partial z>0, \Delta S>0)$; at low latitudes, the atmosphere is more stable or less convective. The same pattern in the vertical temperature

\section{Temperature Variation}

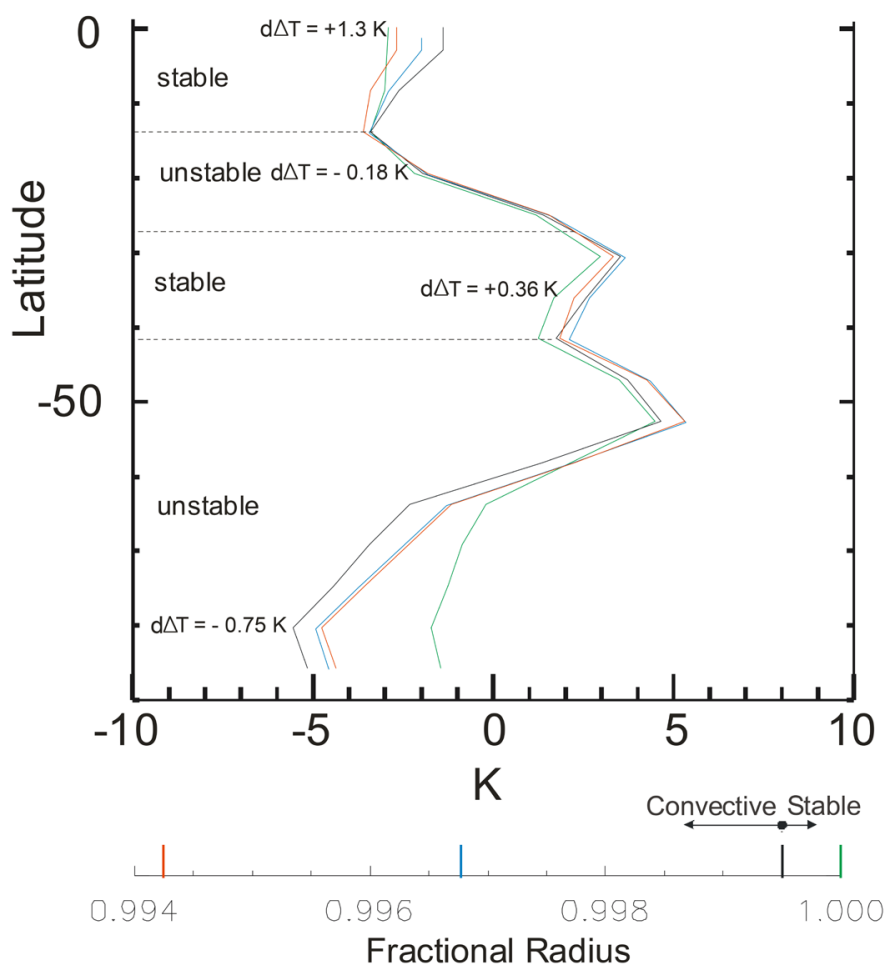

Figure 6. The computed temperatures (Figure 4(b)) relative to the global average, $\Delta T$, are presented on an expanded scale for the southern hemisphere to high-light the variations with latitude (altitudes shown with the fractional (\%) radius scale identified with color code). The temperature differences with altitude, $d \Delta T=\Delta T$ (top-black) $-\Delta T$ (bottom-red), produce an alternating latitude pattern with reduced and enhanced convective instability, where the latitude bands with $d \Delta T<0$ provide dynamical conditions favorable for generating the long lived vortices like the GRS and white/brown ovals. (Figure taken from Chan and Mayr [2].) 
0 ), opposite to the pattern of the equatorial region; the atmosphere is more unstable, more convective. A similar pattern, though much weaker, is also produced in the region around $20^{\circ}$ latitude where the convective instability is amplified $(\Delta S<0)$.

The vertical temperature gradients, $\partial \Delta \mathrm{T} / \partial \mathrm{z}$, across the globe alternate direction to produce distinct latitude bands with enhanced convective instability, where long-lived cyclones can form like the GRS and white/brown ovals in the region around $20^{\circ}$ latitude. The convective instability is largest and most prominent at high latitudes where circumpolar cyclones are observed from the Juno spacecraft (e.g. Tabataba-Vakili et al. [10]).

\section{Conclusions}

The present review was stimulated by a recent conference paper about a numerical simulation addressing the longevity of the Great Red Spot (Hassanzadeh and Marcus [33]). In this model, the imposed vertical motions from the meridional circulation are quoted as the key for extending the life time of the Great Red Spot (GRS).

On a similar track, Mayr et al. [1] proposed that the vertical velocities in the global scale meridional circulation can produce the observed latitude bands where the long-lived Jovian vortices are mainly generated. Following Kuiper [23], the GRS and white/brown ovals are treated like terrestrial hurricanes. In this analysis, the depths and life-times of the vortices were estimated (Holton [26] [27]) to produce spin-down times of 2 years for the GRS, and 3 months for the white/brown ovals. Discovered centuries ago, the relatively long life time of the GRS indicates that it must reside deep inside Jupiter's convection region. And the same applies to the white and brown ovals, which have life-times far exceeding their spin-down times. Numerical experiments show that long-lived vortices can be spontaneously generated inside the fast rotating Jovian type convection zone (Chan and Mayr [22]).

The question is what generates the latitude bands with enhanced convective instability where the white and brown ovals are observed and in broad terms, the answer can be provided by the mulita-cellular meridional circulation illustrated in Figure 3. As illustrated in Figure 2(a) more closely, the upward motions in the meridional circulation around the equator, for example, supply energy from below and increase the temperature and stability. In that environment, the convective instability is suppressed, so that the dynamical conditions are not favorable for generating hurricane like vortices. On the other hand near $20^{\circ}$ latitude for example, illustrated in Figure 2(b), the downward motions in the meridional circulation transport the energy to lower altitudes to increase the convective instability. Under such conditions, the dynamical environment is favorable for generating and sustaining the GRS and the white/brown ovals.

The proposed mechanism for the formation of latitude bands with variable convective instability is supported by numerical results from a spectral model 
that simulates the global variations of the Jovian atmosphere (Chan and Mayr [2]). Generated by the internal planetary energy source, the resulting variations of the temperature and zonal winds are shown in Figure 4 for the convective model atmosphere. In geostrophic balance, the alternating zonal winds are generated by the latitudinal variations of pressure and related temperature. And the temperature variations in turn are dissipated and transformed by the global scale meridional circulation.

In Figure 6 are shown the computed temperature perturbations relative to the global average, $\Delta T$, presented on an expanded scale for the southern hemisphere to high-light the temperature differences with altitude, $d \Delta T$, the measure of convective instability. It shows that the temperature increases with altitude around the equator. The temperature difference between the top (black) and bottom (red) of the convective atmosphere is positive, $d \Delta T>0$; the atmosphere is more stable, less convective. And the same kind of temperature variation is produced at latitudes around $35^{\circ}$. But at high latitudes, and to lesser extent around $20^{\circ}$, the temperature perturbations decrease with altitude, $d \Delta T<0$; the atmosphere is less stable, more convective. Generated by the downward motions in the meridional circulation (Figure 5), latitude bands are generated with amplified convective instability, where the dynamical conditions are more favorable for generating Jovian type vortices.

On Earth, the hurricanes are spawned in the tropics after the summer season with elevated ocean temperatures when the upper troposphere is turning colder to increases the convective instability, analogous to the temperature decrease produced by the meridional circulation that stimulates the Jovian vortex.

\section{Acknowledgements}

Funded by The Science and Technology Development Fund, Macau SAR (0045/2018/AFJ). This work was supported by the State Key Laboratory for Lunar and Planetary Sciences, Macau University of Science and Technology. The authors are grateful for the reviewer's comments with the focus on Juno spacecraft observations.

\section{Conflicts of Interest}

The authors declare no conflicts of interest regarding the publication of this paper.

\section{References}

[1] Mayr, H.G., Maeda, K. and Harris, I. (1985) Conjecture about a Hurricane System in the Jovian Atmosphere. Earth Moon Planets, 32, 183-192. https://doi.org/10.1007/BF00054175

[2] Chan, K.L. and Mayr, H.G. (2008) A Shallow Convective Model for Jupiter's Alternating Wind Bands. Journal of Geophysical Research, 113, E10002.

https://doi.org/10.1029/2008JE003124

[3] Smith, B.A., Soderblom, L.A., Johnson, T.V., Ingersoll, A.P., Collins, S.A., Shoe- 
maker, E.M., Hunt, G.E., Masursky, H., Carr, M.H., Davies, M.E., Cook, A.F., Boyce, J., Danielson, G.E., Owen, T., Sagan, C., Beebe, R.F., Veverka, J., Strom, R.G., McCauley, J.F., Morrison, D., Briggs, G.A. and Suomi, V.E. (1979) The Jupiter System through the Eyes of Voyager 1. Science, 204, 951-972.

https://doi.org/10.1126/science.204.4396.951

[4] Smith, B.A., Beebe, R., Boyce, J., Briggs, G., Carr, M., Collins, S.A., Cook II, A.F., Danielson, G.E., Davies, M.E., Hunt, G.E., Ingersoll, A., Johnson, T.V., Masursky, H., McCauley, J., Morrison, D., Owen, T., Sagan, C., Shoemaker, E.M., Strom, R., Suomi, V.E. and Veverka, J. (1979) The Galilean Satellites and Jupiter: Voyager 2 Imaging Science Results. Science, 206, 927-950.

https://doi.org/10.1126/science.206.4421.927

[5] Mitchell, J.L., Beebe, R.F., Ingersoll, A.P. and Garneau, G.W. (1981) Flow Fields within Jupiter's Great Red Spot and White Oval. Journal of Geophysical Research, 86, 8751-8757. https://doi.org/10.1029/JA086iA10p08751

[6] Simon-Miller, A.A., Gierasch, P.J., Beebe, R.F., Conrath, B., Flasar, F.M., Achterberg, R.K. and the Cassini CIRS Team (2002) New Observational Results Concerning Jupiter's Great Red Spot. Icarus, 158, 249-266. https://doi.org/10.1006/icar.2002.6867

[7] Porco, C.C., West, R.A., McEwen, A., Del Genio, A.D., Ingersoll, A.P., Thomas, P., Squyres, S., Dones, L., Murray, C.D., Johnson, T.V., Burns, J.A., Brahic, A., Neukum, G., Veverka, J., Barbara, J.M., Denk, T., Evans, M., Ferrier, J.J., Geissler, P., Helfenstein, P., Roatsch, T., Throop, H., Tiscareno, M. and Vasavada, A.R. (2003) Cassini Imaging of Jupiter's Atmosphere, Satellites, and Rings. Science, 299, 1541-1547. https://doi.org/10.1126/science.1079462

[8] Sánchez-Lavega, A., Hueso, R., Eichstädt, G., Orton, G., Rogers, J., Hansen, C., Momary, T., Tabataba-Vakili, F. and Bolton, S. (2018) The Rich Dynamics of Jupiter's Great Red Spot from JunoCam: Juno Images. The Astronomical Journal, 156, 162. https://doi.org/10.3847/1538-3881/aada81

[9] Galanti, E., Kaspi, Y., Simons, F., Durante, D., Parisi, M. and Bolton, S. (2019) Determining the Depth of Jupiter's Great Red Spot with Juno: A Slepian Approach. The Astronomical Journal Letters, 874, L24. https://doi.org/10.3847/2041-8213/ab1086

[10] Tabataba-Vakili, F., Rogers, J., Eichstaedt, G., Orton, G., Hansen, C., Momary, T., Sinclair, J., Giles, R., Caplinger, M., Ravine, M. and Bolton, S. (2020) Long-Term Tracking of Circumpolar Cyclones on Jupiter from Polar Observations with JunoCam. Icarus, 335, Article ID: 113405. https://doi.org/10.1016/j.icarus.2019.113405

[11] Marcus, P.S. (1988) Numerical Simulation of Jupiter's Great Red Spot. Nature, 331, 693-696. https://doi.org/10.1038/331693a0

[12] Marcus, P.S., Kundu, T. and Lee, C. (2000) Vortex Dynamics and Zonal Flows. Physics of Plasmas, 7, 1630-1640. https://doi.org/10.1063/1.874045

[13] Marcus, P.S., Pei, S., Jiang, C.H. and Hassanzadeh, P. (2013) Three Dimensional Vortices Generated by Self-Replication in Stably Stratified Rotating Shear Flows. Physical Review Letters, 111, Article ID: 084501. https://doi.org/10.1103/PhysRevLett.111.084501

[14] Showman, A.P., Gierasch, P.J. and Lian, Y. (2006) Deep Zonal Winds Can Result from Shallow Driving in a Giant-Planet Atmosphere. Icarus, 182, 513-526. https://doi.org/10.1016/j.icarus.2006.01.019

[15] Showman, A.P. (2007) Numerical Simulations of Forced Shallow-Water Turbulence: Effects of Moist Convection on the Large-Scale Circulation of Jupiter and Sa- 
turn. Journal of the Atmospheric Sciences, 64, 3132-3157. https://doi.org/10.1175/JAS4007.1

[16] Mayr, H.G., Harris, I. and Chan, K.L. (1984) Differential Rotation in a Solar-Driven Quasi-Axisymmetric Circulation. Earth Moon Planets, 30, 245-274.

https://doi.org/10.1007/BF00056202

[17] Ingersoll, A.P., Gierasch, P.J., Banfield, D. and Vasavada, A.R. (2000) Galileo Imaging Team: Moist Convection as an Energy Source for the Large-Scale Motions in Jupiter's Atmosphere. Nature, 403, 630-632. https://doi.org/10.1038/35001021

[18] Zhang, K. and Schubert, G. (2000) Tele-Convection: Remotely Driven Thermal Convection in Rotating Stratified Spherical Layers. Science, 290, 1944.

https://doi.org/10.1126/science.290.5498.1944

[19] Christensen, U.R. (2001) Zonal Flow Driven by Deep Convection in the Major Planets. Geophysical Research Letters, 28, 2553-2556. https://doi.org/10.1029/2000GL012643

[20] Aurnou, J.M. and Olson, P.I. (2001) Strong Zonal Winds from Thermal Convection in a Rotating Spherical Shell. Geophysical Research Letters, 28, 2557-2559. https://doi.org/10.1029/2000GL012474

[21] Heimpel, M. and Aurnou, J. (2007) Turbulent Convection in a Rapidly Rotating Spherical Shell: A Model for Equatorial and High Latitude Jets on Jupiter and Saturn. Icarus, 187, 540-557. https://doi.org/10.1016/j.icarus.2006.10.023

[22] Chan, K.L. and Mayr, H.G. (2013) Numerical Simulation of Convectively Generated Vortices: Application to the Jovian Planets. Earth and Planetary Science Letters, 71, 212-219. https://doi.org/10.1016/j.epsl.2013.03.046

[23] Kuiper, G.P. (1972) Lunar and Planetary Laboratory Studies of Jupiter. Sky and Telescope, 43, 75-81.

[24] Charney, J.G. and Eliassen, A. (1964) On the Growth of the Hurricane Depression. Journal of the Atmospheric Sciences, 21, 68-75. https://doi.org/10.1175/1520-0469(1964)021<0068:OTGOTH >2.0.CO;2

[25] Ooyama, K. (1969) Numerical Simulation of the Life Cycle of Tropical Cyclones. Journal of the Atmospheric Sciences, 26, 3-40. https://doi.org/10.1175/1520-0469(1969)026<0003:NSOTLC $>2.0 . C O ; 2$

[26] Holton, J.R. (1975) The Dynamic Meteorology of the Stratosphere and Mesosphere. American Meteorological Society, Meteorological Monograph, Volume 15, Issue 37, Boston. https://doi.org/10.1007/978-1-935704-31-7

[27] Holton, J.R. (1979) An Introduction to Dynamic Meteorology. Academic Press, New York.

[28] Hanel, R., Conrath, B., Flaser, M., Kunde, V., Lowman, P., Maguire, W., Pearl, J., Pirraglia, J., Samuelson, R., Gautier, D., Gierasch, P., Kumar, S. and Ponnamperuma, C. (1979) Infrared Observations of the Jovian System from Voyager 1. Science, 204, 972-976. https://doi.org/10.1126/science.204.4396.972-a

[29] Hanel, R., Conrath, B., Flaser, M., Herath, L., Lia, V., Samuelson, R., Gautier, D., Gierasch, P., Horn, L., Kumar, S. and Ponnamperuma, C. (1979) Infrared Observations of the Jovian System from Voyager 2. Science, 206, 952-956. https://doi.org/10.1126/science.206.4421.952

[30] Mayr, H.G. and Harris, I. (1983) Quasi-Axisymmetric Circulation and Superrotation in Planetary Atmospheres. Astronomy \& Astrophysics, 121, 124-136.

[31] Chan, K.L., Mayr, H.G., Mengel, J.G. and Harris, I. (1994) A “Stratified” Spectral Model for Stable and Convective Atmospheres. Journal of Computational Physics, 
113, 165-176. https://doi.org/10.1006/jcph.1994.1128

[32] Chan, K.L. and Mayr, H.G. (2008) Convective Models of Jupiter's Wind Bands: Transition from Deep to Shallow Envelopes. 40 th Annual Meeting of Division for Planetary Sciences of American Astronomical Society, Ithaca, 10-15 October 2008.

[33] Hassanzadeh, P. and Marcus, P.S. (2013) On the Unexpected Longevity of the Great Red Spot, Oceanic Eddies, and Other Baroclinic Vortices. Abstract of the American Physical Society's Division of Fluid Dynamics Meeting, Pittsburgh, 24-26 November 2013. 